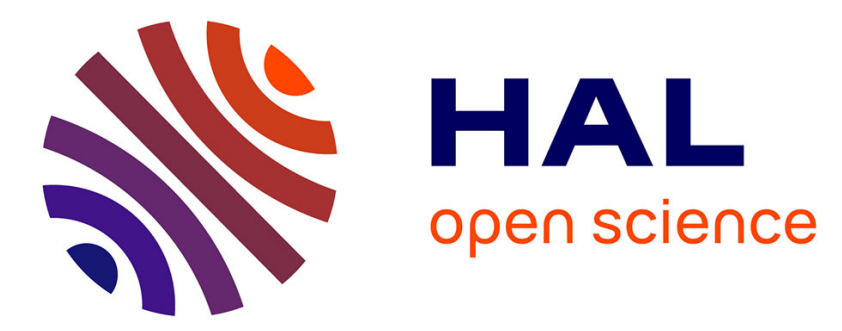

\title{
A simple method to assess group difference in RT-qPCR reference gene selection using GeNorm: The case of the placental sex
}

\author{
Joey St-Pierre, Jean-Charles Grégoire, Cathy Vaillancourt
}

\section{- To cite this version:}

Joey St-Pierre, Jean-Charles Grégoire, Cathy Vaillancourt. A simple method to assess group difference in RT-qPCR reference gene selection using GeNorm: The case of the placental sex. Scientific Reports, 2017, 7 (1), pp.16923. 10.1038/s41598-017-16916-y • pasteur-01854622

\section{HAL Id: pasteur-01854622}

https://hal-riip.archives-ouvertes.fr/pasteur-01854622

Submitted on 6 Aug 2018

HAL is a multi-disciplinary open access archive for the deposit and dissemination of scientific research documents, whether they are published or not. The documents may come from teaching and research institutions in France or abroad, or from public or private research centers.
L'archive ouverte pluridisciplinaire HAL, est destinée au dépôt et à la diffusion de documents scientifiques de niveau recherche, publiés ou non, émanant des établissements d'enseignement et de recherche français ou étrangers, des laboratoires publics ou privés.

\section{(c)(1)}

Distributed under a Creative Commons Attribution| 4.0 International License 


\section{SCIENTIFIC REP RTS}

Received: 26 June 2017

Accepted: 20 November 2017

Published online: 05 December 2017
A simple method to assess group difference in RT-qPCR reference gene selection using GeNorm: The case of the placental sex

\author{
Joey St-Pierre ${ }^{1,2}$, Jean-Charles Grégoire ${ }^{3}$ \& Cathy Vaillancourt $\mathbb{1}^{1,2}$
}

Normalization with proper reference genes is a crucial step in obtaining accurate mRNA expression levels in RT-qPCR experiments. GeNorm and NormFinder are two commonly used software packages that help in selecting the best reference genes, based on their expression stability. However, GeNorm does not take into account a group variable, such as sample sex, in its calculation. We demonstrate a simple calculation step to assess the variability of such parameters by multiplying the GeNorm M value with the difference of $\mathrm{Cq}$ values between groups. To test this, we used 28 reference gene candidates, to analyze 20 placental samples ( 10 of each sex), and by using HPRT1 (lower Cq values in male placentas $(P=0.017))$, as a target gene. Our calculation demonstrates that the RPL30-GAPDH reference gene combination is the better option to assess small placental sex differences in mRNA level, versus the selection obtained from GeNorm or NormFinder. The HPRT1 normalized mRNA expression level is different between placental sexes, using RPL30 and GAPDH as reference genes $(P=0.01)$, but not when using genes suggested by GeNorm or NormFinder. These results indicate that the proposed calculation is appropriate to assess small variations in mRNA expression between 2 groups.

Reverse transcription quantitative polymerase chain reaction (RT-qPCR) is an efficient tool to assess mRNA level. However, the production of reliable results requires several steps to be followed, which has not always been the case in some publications using RT-qPCR results ${ }^{1,2}$. An important step is the selection of stable mRNA reference genes, in order to achieve accurate normalization ${ }^{3}$.

Poor reference gene selection can lead to inaccurate results ${ }^{4}$. Reference genes are often described as housekeeping genes that are necessary for regular cell function. These genes are expected to have a stable mRNA expression level throughout all cell types and conditions tested ${ }^{5}$. However, this is rarely the case as diseases, treatments or experimental conditions affect the stability of commonly used reference genes ${ }^{6}$. This indicates the need to test for reference gene variability between treatments or sample conditions, for all experiments. Several software options are widely utilized to assess the variability of reference genes, including GeNorm and NormFinder for qPCR experiment ${ }^{3,4}$. While NormFinder offers a method of reference genes selection that takes into account intra- and inter-group variability, such as sex, GeNorm does not differentiate between groups of samples or treatments ${ }^{4,7}$.

Sample sex is an important parameter for biological and molecular biology research, with grant funding bodies and publishers requiring sample sex to be evaluated or clear reasons for its exclusion ${ }^{8-11}$. Placental research is an area where fetal sex differences are of significant importance ${ }^{12-14}$, including in regard to placental adaptation strategy ${ }^{12,15,16}$. Unfortunately, placental/fetal sex is not always a universally adopted parameter ${ }^{8}$.

Reference gene selection is especially important when working with tissue samples or primary cell lines. This study demonstrates a new method of calculation for the selection of reference genes when assessing the difference between two groups, exemplified in this case by placental sex.

${ }^{1}$ INRS-Centre Institut Armand-Frappier and BioMed Research Center, Laval, Canada. ${ }^{2}$ Center for Interdisciplinary Research on Well-Being, Health, Society and Environment, Université du Québec à Montréal, Montréal, OC, Canada. ${ }^{3}$ INRS-Centre Énergie Matériaux Télécommunications, Montréal, QC, Canada. Correspondence and requests for materials should be addressed to C.V. (email: cathy.vaillancourt@iaf.inrs.ca) 


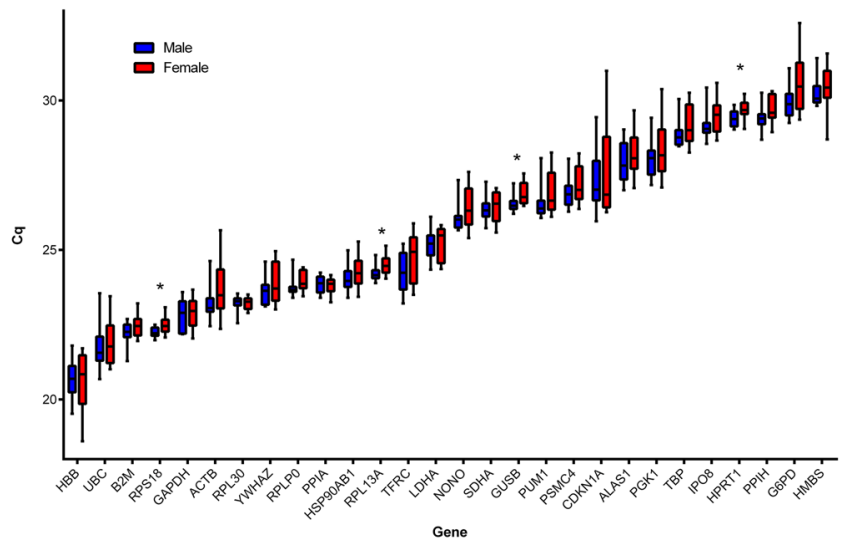

Figure 1. Distribution of the Cq values obtained for 28 candidate reference genes, from the reference genes H96 and $\mathrm{H} 96$ plus primePCR plates (Bio-Rad, see Table 1) for 10 placental samples for each fetal sex. Boxes represent the median Cq values with interquartile ranges representing minimum value to maximum value. Student's T tests were performed on Cq values between placental sexes. *P $\leq 0.05 . \mathrm{n}=20$ placentas (10 males; 10 females).

\begin{tabular}{|c|c|c|}
\hline Gene symbol & Name & RefSeq \\
\hline ACTB & Beta Actin & NM_001101.3 \\
\hline ALAS1 & Aminolevulinate, delta-, synthase 1 & NM_000688.5 \\
\hline $\mathrm{B} 2 \mathrm{M}$ & Beta-2-microglobulin & NM_004048.2 \\
\hline CDKN1A & Cyclin-dependent kinase inhibitor 1A (p21, Cip1) & NM_000389.4 \\
\hline G6PD & Glucose-6-phosphate dehydrogenase & NM_000402.4 \\
\hline GAPDH & Glyceraldehyde-3-phosphate dehydrogenase & NM_001256799.2 \\
\hline GUSB & Glucuronidase, beta & NM_000181.3 \\
\hline HBB & Hemoglobin, beta & NM_000518.4 \\
\hline HMBS & Hydroxymethylbilane synthase & NM_001024382.1 \\
\hline HPRT1 & Hypoxanthine phosphoribosyltransferase 1 & NM_000194.2 \\
\hline HSP90AB1 & Heat shock protein $90 \mathrm{kDa}$ alpha (cytosolic), class B member 1 & NM_001271969.1 \\
\hline IPO8 & Importin 8 & NM_001190995.1 \\
\hline LDHA & Lactate dehydrogenase A & NM_001135239.1 \\
\hline NONO & Non-POU domain containing, octamer-binding & NM_001145408.1 \\
\hline PGK1 & Phosphoglycerate kinase 1 & NM_000291.3 \\
\hline PPIA & Peptidylprolyl isomerase A (cyclophilin A) & NM_001300981.1 \\
\hline PPIH & Peptidylprolyl isomerase $\mathrm{H}$ (cyclophilin $\mathrm{H}$ ) & NM_006347.3 \\
\hline PSMC4 & Proteasome (prosome, macropain) 26S subunit, ATPase, 4 & NM_006503.3 \\
\hline PUM1 & Pumilio RNA-binding family member 1 & NM_001020658.1 \\
\hline RPL13A & Ribosomal protein L13a & NM_001270491.1 \\
\hline RPL30 & Ribosomal protein L30 & NM_000989.3 \\
\hline RPLP0 & Ribosomal protein, large, $\mathrm{P} 0$ & NM_001002.3 \\
\hline RPS18 & Ribosomal protein S18 & NM_022551.2 \\
\hline SDHA & Succinate dehydrogenase complex, subunit A, flavoprotein (Fp) & NM_001294332.1 \\
\hline TBP & TATA box binding protein & NM_001172085.1 \\
\hline TFRC & Transferrin receptor (p90, CD71) & NM_001128148.1 \\
\hline UBC & Ubiquitin $\mathrm{C}$ & NM_021009.6 \\
\hline YWHAZ & $\begin{array}{l}\text { Tyrosine 3-monooxygenase/tryptophan 5-monooxygenase } \\
\text { activation protein, zeta }\end{array}$ & NM_001135699.1 \\
\hline
\end{tabular}

Table 1. List of candidate reference genes included in the reference gene H96 and H96 plus prime PCR plates with RefSeq number.

\section{Results}

Cq results. Figure 1 shows the distribution of $\mathrm{Cq}$ values for 28 reference genes in 20 placental tissues ( 10 for each sex) from PrimePCR Reference Gene H96 and Reference Gene H96 plus plates. Four reference genes showed significantly lower Cq, indicating higher mRNA levels, in male compared to female placentas (RPS18, $\mathrm{p}=0.018 ; R P L 13 A, \mathrm{p}=0.017 ; G U S B, \mathrm{p}=0.015 ; H P R T 1, \mathrm{p}=0.017)$. Results were similar using the absolute quantity of mRNA $\left(2^{\Delta \mathrm{Cq}}\right)$ (data not shown). 

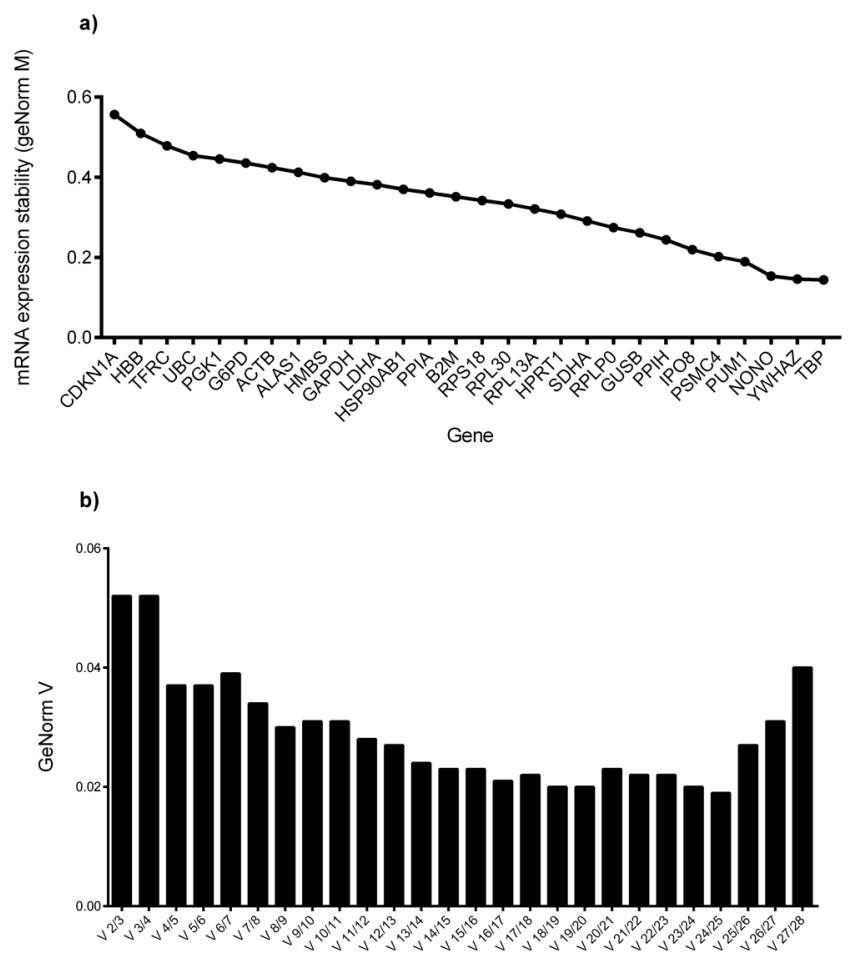

Figure 2. (a) GeNorm M results for all reference genes tested for all the placenta samples taken together. (b) GeNorm V value for all reference genes tested. These results represent the best reference genes selection (lower GeNorm M score) as well as the quantity of reference genes optimal to use found (GeNorm V score below 0.15 ) using the QbasePlus software.

GeNorm and NormFinder. Figure 2a shows the GeNorm M analysis for all 20 placental samples. Lower GeNorm $M$ values represent the most stable reference genes. The GeNorm software package also computes variation of reference genes used for normalization (GeNorm $V: n /(n+1)$, indicating that 2 reference genes are suitable for RT-qPCR normalization in our experiment $(2 / 3$ genes GeNorm $\mathrm{V}<0.15)$ (Fig. $2 b)$. Thus, for all 20 samples, the best reference genes pair according to the GeNorm software is TBP and YWHAZ.

The samples were then split into two sets according to sex, which were entered separately in the GeNorm software package. Results demonstrate that for male placentas, NONO and TBP are the most stable reference genes (Fig. 3a), whilst for female placentas, TBP and $Y W H A Z$ are the most stable (Fig. 3b). Four genes have a highly different GeNorm rank between the sexes: G6PD, ACTB, HMBS and PGK1. The reference gene selection software NormFinder was also used to assess the best reference gene using intra- and inter-group (i.e. placental sex) variation. Results indicate IPO8 and TBP to be the combination of reference genes to utilize for normalization (supplemental data Figure S1).

Novel calculation method. A novel calculation method was used to obtain the best reference genes possible for normalization in the two groups. This calculation requires the multiplication of the GeNorm $\mathrm{M}$ value by the difference of $\mathrm{Cq}$ between the groups $(\Delta \mathrm{Cq} * \mathrm{M})$. The lowest number indicates the genes for which normalization provides the smallest bias for groups, while still accounting for pairwise variation (Fig. 4). Using this calculation on the 20 samples, the RPL30 and GAPDH pair is the best combination of reference genes.

To validate this calculation, HPRT1 was used as a target gene for normalized mRNA level $\left(2^{\triangle \triangle \mathrm{Cq}}\right)$, given that HPRT1 mRNA level is significantly different between males and females. Figure 5 represents the normalized HPRT1 mRNA level, using different reference genes chosen from the results of GeNorm (Fig. 2) and NormFinder (Fig. S1). RPS18 was chosen as a reference gene to show normalizing with a reference gene that has a significant difference between two groups. As expected, normalizing with RPS18 resulted in no significant difference in HPRT1 mRNA level between sexes (Fig. 5). IPO8 and TBP were selected as best reference genes by NormFinder (Fig. S1), while TBP and $Y W H A Z$ are the two best reference genes obtained with the GeNorm calculation for our samples (Fig. 2a). However, when normalizing with either IPO8 and TBP or TBP and YWHAZ, there was no significant difference between males and females for placental HPRT1 mRNA level (Fig. 5). When RPL30 and the $R P L 30+G A P D H$ combination were used as reference genes, a significantly higher relative HPRT1 mRNA level was obtained in male placentas ( $\mathrm{p}=0.009$ and $\mathrm{p}=0.010$, respectively; Fig. 5). Results were similar for the other genes that have a significantly different absolute mRNA levels between placental sexes (RPS18, GUSB and RPL13A genes, data not shown). However, when normalizing every other gene with the RPL30 and GAPDH combination, no statistically significant difference was evident between male and female placentas (data not shown). This indicates that the new calculation method proposed here for choosing the best reference genes, did not result in "false positive" group differences after normalization. 

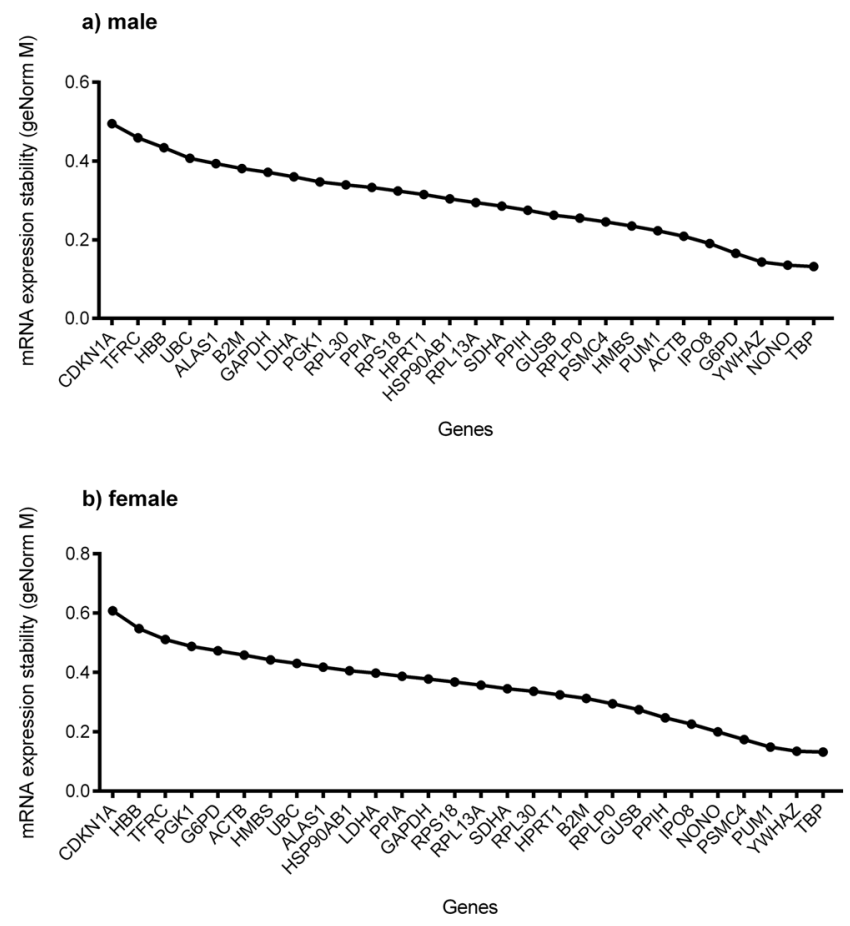

Figure 3. GeNorm results for (a) male placentas and (b) female placentas. Ten placentas from each sex were used to obtain results with PrimePCR Reference Gene H96 and Reference Gene H96 Plus plates (Bio-Rad). Cq values were transferred into the Qbase Plus software (Biogazelle) to obtain GeNorm M values.

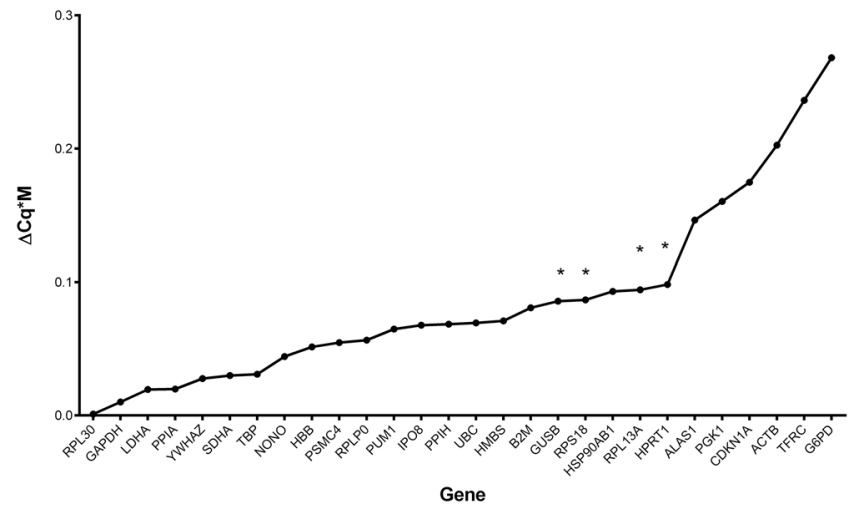

Figure 4. Results from multiplication of the GeNorm $M$ value with the difference of average of Cq results between placental sexes $(\Delta \mathrm{Cq})$. A smaller $\Delta \mathrm{Cq} * \mathrm{M}$ score represents the optimal value for gene expression results normalization for two groups (placental sex). *Genes showing significantly different Cq values between male and female placentas (Student's T test, $\mathrm{P} \leq 0.05$; see Fig. 1).

\section{Discussion}

In this study, we demonstrate the importance of placental sex in reference gene selection and that proper reference genes selection is crucial to obtaining accurate results. The results here show that placental sex needs to be taken into consideration in the selection of reference genes for the normalization of placental mRNA expression levels. Significant Cq differences in placental mRNA levels were evident in some of the most commonly used reference genes: HPRT1, GUSB, RPL13A and RPS18. These genes had significantly lower Cq values for male, versus female, placentas, indicating that their utilization for gene normalization would produce biased results, both inappropriate non-significant and significant results ${ }^{1,2,4,17}$.

Cvitic et al. found that most of the placental mRNA level of genes tested were higher in boy placentas than in girl placentas in microarray analysis, but this varied greatly between cell types ${ }^{18}$. This team used HPRT1 as reference gene, but in their analysis, HPRT1 did not vary between samples sexes. HPRT1 is a gene that is present on the chromosome X. The HPRT1 gene is inactivated in early embryology in female fetuses and has already been shown to have significantly different expression in regard to sample sex in other tissues ${ }^{19-21}$. In our study, mRNA levels for GUSB, RPL13A, RPS18 and HPRT1 are significantly higher in boy placentas than in girl placentas (lower 


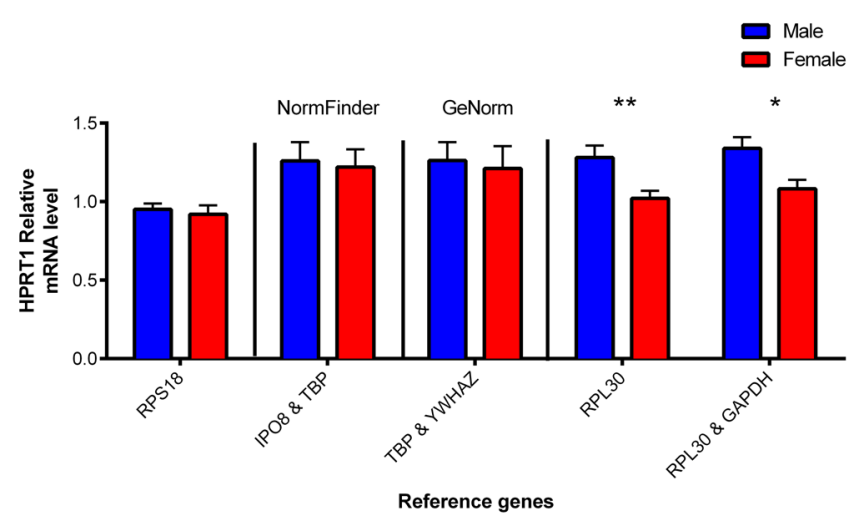

Figure 5. HPRT1 mRNA expression levels normalized with different reference genes in human placenta. RPS18 has significantly different Cq values between sexes. IPO \& TBP were selected using NormFinder software. TBP \& $Y W H A Z$ were selected using GeNorm software. RPL30 and RPL30\& GAPDH were selected using the $\triangle \mathrm{Cq}$ form the placental sex multiplied by GeNorm $M$ value. ${ }^{*} \mathrm{p} \leq 0.05$; $* * \mathrm{p} \leq 0.01 \mathrm{n}=10$ per placental sex.

$\mathrm{Cq}$ ) and we did not observe any reference gene mRNA levels that were higher in girl (as opposed to boy) placentas. These could be explained by the fact that the RNA taken in our study was obtained from the trophoblastic layer of the placenta that has a generally higher overall gene expression in male placentas compared to female placentas $^{14,18}$.

It is noteworthy that $Y W H A Z$ was seen expressed significantly higher in male placentas compared to female placentas by Cleal et al. ${ }^{14}$ but not in our study. This could be explained by the fact that the authors tested 102 placentas compared to 20 placentas from our study, perhaps leading to a sampling bias resulting from less placental samples ${ }^{14}$. Another difference between their study compared to ours is that the sampling method might be different as the method used by Cleal et al. is not specified. However, the inconsistency between Cleal et al. results and ours highlights the importance to test and select reference genes for their difference in Cq values for every sample group in every experiment and not to rely only on previous literature.

Choosing a reference gene that does not vary between groups is essential for accurate RT-qPCR results, and this can be applied to placental sex as a subgroup ${ }^{7}$. In this study, we tested 28 different candidate reference genes for 20 human placental samples and rated them from best to worst using two of the most popular methods. Yet, when normalizing with the reference genes combinations found by commonly used software packages, there was no significant difference in HPRT1 mRNA level between male and female placentas. This would suggest that NormFinder's reference gene selection does not include proper correction for the difference in the average gene expression, at least in our experiment.

When using reference genes selected by our new method of calculation, we observed a significant difference in the placental mRNA level of HPRT1 between male and female placentas, that reflects the significant difference observed for the Cq values and for the absolute mRNA expression. The same is also observed for all genes which have significantly different $\mathrm{Cq}$ values between male and female populations. Moreover, using our calculation and in accordance with the Cq values obtained, we did not identify other significant differences in mRNA levels between sexes other than those that were significantly different for their Cq values and absolute mRNA level (GUSB, RPL13A and RPS18). This would mean that, when comparing for different groups (or in our case placental sexes), multiplying GeNorm $M$ results by the Cq difference between groups will enable to find reference genes that will provide a more accurate normalization for group comparison, while not obtaining false-positive results.

However, this simple tool should be used with caution and does not replace a researcher's judgement, as is the case with any other method of selecting reference genes for RT-qPCR. Furthermore, the calculations we propose might not fit all situations encountered as they have only been tested human placentas and only for groups based on fetal sexes.

\section{Conclusion}

The main finding in this study is that even though the genes with the best GeNorm $M$ values could be used to normalize RT-qPCR Cq values, this would not take into account group differences and might conceal slight differences between groups when normalizing results. While NormFinder does take into account a sample group such as placental sex, results using either GeNorm's or NormFinder's suggested genes did not provide the best reference gene to assess small mRNA level differences between samples. Here we have introduced a novel method of calculation for finding the best reference genes for normalization between groups. While this method is not perfect by any means, it is straightforward to use and can help pinpoint genes that have lower mRNA average variability between groups as well as the most stable in the pairwise variation method. The results presented in this study also highlights the importance of proper reference gene selection as well as the importance of not only relying blindly on software calculation results for such a critical part of the RT-qPCR technique. Thus, it is up to the researcher to verify and test differences between the mRNA levels of groups tested with raw RT-qPCR data. 


\begin{abstract}
Materials and Methods
Placental samples. Human term placentas (10 males/10 females; $38-41$ gestation weeks) were obtained immediately after spontaneous vaginal deliveries from uncomplicated singleton pregnancies. Placentas were obtained with informed patient consent and approval of ethical committees at the CHUM-St-Luc Hospital (Montreal, QC) and INRS (Quebec, QC). Women having pathologies, smokers or under medication were excluded. All the experiments were performed according to ethical guidelines.

All samples were processes within $30 \mathrm{~min}$ of placental delivery. Placental tissue collection was realized using methods consistent with the recommendations ${ }^{22}$. Biopsies were taken, guided by a stereological grid, as described previously $^{23}$. As the placenta is a heterogeneous tissue, it is necessary to reduce the sampling site-driven gene expression biases, which is achieved by multistage unbiased random sampling, thereby giving all sites equal possibility of selection. To maximize the representativeness of the extracted RNA, 5 tissue samples were collected from each placenta, using a stratified random sampling method, and then pooled for RNA extraction. The trophoblastic samples were then flash-frozen and kept at $-80^{\circ} \mathrm{C}$ until analysis. All tissue samples were then pooled and ground into powder using a mortar and pestle, kept at very low temperature by dry ice and liquid nitrogen. Placental tissue powders, 15 to $20 \mathrm{mg}$, were weighed using a $1.5 \mathrm{ml}$ tube cooled in liquid nitrogen for RNA isolation. Such steps ensured that the placental samples were not thawed prior to RNA extraction.
\end{abstract}

RNA isolation and cDNA synthesis. The RNA was isolated using the AllPrep DNA/RNA/Protein mini kit (Qiagen, Toronto, ON), after using Qiashredder spin columns (Qiagen) to further disrupt placental tissue, according to the manufacturer's instructions. RNA concentration and purity were assessed using the ND-1000 Nanodrop Spectrophotometer (Thermo Scientific, Waltham, MA). RNA integrity was analyzed using the Experion automated electrophoresis system (Bio-Rad, Hercules, CA). Samples utilized had an RNA quality index (RQI) above 7.5. The cDNA was obtained using the iScript reverse transcription supermix for the RT-qPCR kit (Bio-Rad), following the manufacturer's instructions, for a total of $500 \mathrm{ng}$ of RNA. The samples were then diluted $1 / 20$ in RNase Free Water and stored at $-20^{\circ} \mathrm{C}$ until further analysis.

qPCR. The reference genes analyzed originated from PrimePCR Reference genes H96 and Reference Genes Plus H96 (Bio-Rad). These plates come with 14 different reference gene primers, lyophilized in wells (28 genes; Table 1), as well as controls for positive and negative PCR reaction, reverse transcription control and two RNA integrity controls. The primer sequence is not available for the genes in PrimePCR assays, but the validation data is available online for each gene. The reagent used for qPCR reaction was SsoAdvanced SYBR Green Supermix (Bio-Rad). The reaction was performed according to the manufacturer's instructions, on a CFX96 real-time PCR detection system (Bio-Rad). Assays were performed in triplicate to control for technical errors.

Data analysis. To analyze the reference genes' mRNA expression stability, the qBase + software package (BioGazelle, Zwijnaarde, Belgium) was used to obtain GeNorm results. The qBase + software package calculates gene expression stability (GeNorm $M$ ), as well as the variation from using $n$ reference gene or $n+1$ reference genes to evaluate the lowest number of genes required for accurate normalization (GeNorm V). Statistical analyses were carried out using PRISM 5.0 (Graphpad software, La Jolla, CA). Variance was tested between sexes and normal sample distribution was also assessed, prior to using Student's t-test to evaluate the difference in mRNA level between male and female placentas.

\title{
References
}

1. Bustin, S. A. et al. The MIQE guidelines: Minimum information for publication of quantitative real-time PCR experiments. Clinical Chemistry 55, 611-622, https://doi.org/10.1373/clinchem.2008.112797 (2009).

2. Taylor, S., Wakem, M., Dijkman, G., Alsarraj, M. \& Nguyen, M. A practical approach to RT-qPCR-Publishing data that conform to the MIQE guidelines. Methods 50, S1-S5, https://doi.org/10.1016/j.ymeth.2010.01.005 (2010).

3. Derveaux, S., Vandesompele, J. \& Hellemans, J. How to do successful gene expression analysis using real-time PCR. Methods 50, 227-230, https://doi.org/10.1016/j.ymeth.2009.11.001 (2010).

4. Lanoix, D. et al. Quantitative PCR Pitfalls: The Case of the Human Placenta. Molecular Biotechnology, 1-10, https://doi.org/10.1007/ s12033-012-9539-2 (2012).

5. Eisenberg, E. \& Levanon, E. Y. Human housekeeping genes, revisited. Trends in genetics: TIG 29, 569-574, https://doi.org/10.1016/j. tig.2013.05.010 (2013).

6. Kozera, B. \& Rapacz, M. Reference genes in real-time PCR. Journal of Applied Genetics 54, 391-406, https://doi.org/10.1007/s13353013-0173-x (2013).

7. Andersen, C. L., Jensen, J. L. \& Orntoft, T. F. Normalization of real-time quantitative reverse transcription-PCR data: a model-based variance estimation approach to identify genes suited for normalization, applied to bladder and colon cancer data sets. Cancer research 64, 5245-5250, https://doi.org/10.1158/0008-5472.can-04-0496 (2004).

8. Ritz, S. A. et al. First steps for integrating sex and gender considerations into basic experimental biomedical research. The FASEB Journal, https://doi.org/10.1096/fj.13-233395 (2013).

9. Heidari, S., Babor, T. F., De Castro, P., Tort, S. \& Curno, M. Sex and Gender Equity in Research: rationale for the SAGER guidelines and recommended use. Research Integrity and Peer Review 1, 2, https://doi.org/10.1186/s41073-016-0007-6 (2016).

10. Institute of Medicine Board on Population, H. \& Public Health, P. In Sex-Specific Reporting of Scientific Research: A Workshop Summary (National Academies Press (US)National Academy of Sciences., 2012).

11. Del Boca, F. K. Addressing sex and gender inequities in scientific research and publishing. Addiction (Abingdon, England) 111, 1323-1325, https://doi.org/10.1111/add.13269 (2016).

12. Clifton, V. L. Review: Sex and the Human Placenta: Mediating Differential Strategies of Fetal Growth and Survival. Placenta 31, S33-S39, https://doi.org/10.1016/j.placenta.2009.11.010 (2010).

13. Adibi, J. et al. IFPA meeting 2016 workshop report II: Placental imaging, placenta and development of other organs, sexual dimorphism in placental function and trophoblast cell lines. Placenta, https://doi.org/10.1016/j.placenta.2017.02.021 (2017).

14. Cleal, J. K., Day, P. L., Hanson, M. A. \& Lewis, R. M. Sex differences in the mRNA levels of housekeeping genes in human placenta. Placenta 31, 556-557, https://doi.org/10.1016/j.placenta.2010.03.006 (2010).

15. Osei-Kumah, A., Smith, R., Jurisica, I., Caniggia, I. \& Clifton, V. L. Sex-specific differences in placental global gene expression in pregnancies complicated by asthma. Placenta 32, 570-578, https://doi.org/10.1016/j.placenta.2011.05.005 (2011). 
16. Duran Erdolu, M., Kosus, A., Kosus, N., Dilmen, G. \& Kafali, H. Relationship between placental localisation, birth weight, umbilical Doppler parameters, and foetal sex. Turkish journal of medical sciences 44, 1114-1117 (2014).

17. Wang, H.-L. et al. Evaluation of Appropriate Reference Genes for Reverse Transcription-Quantitative PCR Studies in Different Tissues of a Desert Poplar via Comparision of Different Algorithms. International journal of molecular sciences 16, 20468-20491, https://doi.org/10.3390/ijms160920468 (2015).

18. Cvitic, S. et al. The Human Placental Sexome Differs between Trophoblast Epithelium and Villous Vessel Endothelium. PLoS ONE 8, e79233, https://doi.org/10.1371/journal.pone.0079233 (2013).

19. Graves, J. A. Review: Sex chromosome evolution and the expression of sex-specific genes in the placenta. Placenta 31(Suppl), S27-32, https://doi.org/10.1016/j.placenta.2009.12.029 (2010).

20. Verma, A. S. \& Shapiro, B. H. Sex-dependent expression of seven housekeeping genes in rat liver. Journal of gastroenterology and hepatology 21, 1004-1008, https://doi.org/10.1111/j.1440-1746.2005.03948.x (2006).

21. Lucas, E. S. et al. Tissue-specific selection of reference genes is required for expression studies in the mouse model of maternal protein undernutrition. Theriogenology 76, 558-569, https://doi.org/10.1016/j.theriogenology.2011.03.008 (2011).

22. Burton, G. J. et al. Optimising sample collection for placental research. Placenta 35, 9-22, https://doi.org/10.1016/j. placenta.2013.11.005 (2014).

23. Mayhew, T. M. Stereology and the placenta: where's the point?-a review. Placenta 27(Suppl A), S17-25, https://doi.org/10.1016/j. placenta.2005.11.006 (2006).

\section{Acknowledgements}

This study was financially supported by grant from Natural Sciences and Engineering Research Council of Canada (NSERC grant no. 262011-2009) and from the March of Dimes Social and Behavioral Sciences Research (grant no. 12-FY12-179) awarded to CV. JSTP was supported by the Fond de Recherche du Québec en santé (FRQS). The authors would like to thank Dr. Laetitia Laurent for her help in manuscript revision.

\section{Author Contributions}

J. St-Pierre and C. Vaillancourt participated in study conception and design as well as revising critically the manuscript. J. St-Pierre drafted the manuscript and did the experimental work and the data acquisition, and did the statistical analysis. J. St-Pierre, J.-C. Grégoire and C. Vaillancourt contributed to the analysis and interpretation of data. All authors have approved the final version of the article.

\section{Additional Information}

Supplementary information accompanies this paper at https://doi.org/10.1038/s41598-017-16916-y.

Competing Interests: The authors declare that they have no competing interests.

Publisher's note: Springer Nature remains neutral with regard to jurisdictional claims in published maps and institutional affiliations.

Open Access This article is licensed under a Creative Commons Attribution 4.0 International License, which permits use, sharing, adaptation, distribution and reproduction in any medium or format, as long as you give appropriate credit to the original author(s) and the source, provide a link to the Creative Commons license, and indicate if changes were made. The images or other third party material in this article are included in the article's Creative Commons license, unless indicated otherwise in a credit line to the material. If material is not included in the article's Creative Commons license and your intended use is not permitted by statutory regulation or exceeds the permitted use, you will need to obtain permission directly from the copyright holder. To view a copy of this license, visit http://creativecommons.org/licenses/by/4.0/.

(C) The Author(s) 2017 